\title{
CERTAIN WEIL ALGEBRAS OF INFINITE-DIMENSIONAL LIE ALGEBRAS
}

\author{
By Toshikazu Natsume
}

\section{Introduction}

It was shown by $\mathrm{H}$. Cartan [1] that to any finite-dimensional Lie algebra $g$ an acyclic differential graded algebra $W(\mathrm{~g})$ can be assigned, which is called the Weil algebra of $\mathrm{g}$. According to the Chern-Weil theory, if $\mathrm{g}$ is the Lie algebra of a compact connected Lie group $G$, then the cohomology of the subalgebra consisting of basic elements of $W(\mathfrak{g})$ has a geometric interpretation as the universal characteristic classes for the principal $G$-bundles.

Recently, the cohomology theory of infinite-dimensional Lie algebras has been developed from several aspects. For instance, I. M. Gel'fand and D. B. Fuks have investigated the cohomology of the Lie algebra of vector fields on a manifold. In this context, it was shown by C. Godbillon [2] that the cohomology of the Lie algebra of formal vector fields is isomorphic to the cohomology of a truncated Weil algebra $\hat{W}\left(\mathfrak{g l}_{n}\right)$, where $\mathfrak{g l}_{n}$ is the Lie algebra of the general linear group. This result seems to suggest that, in some cases, even for infinite-dimensional Lie algebras the consideration of the Weil algebras will come to an object of our investigation.

The present paper is our first attempt in this direction. We shall mainly consider the Lie algebra $a_{n}$ of formal vector fields and try to calculate the cohomology of a subalgebra consisting of certain basic elements of $W\left(\mathfrak{a}_{n}\right)$. Our main result is stated as follows:

THEOREM 2.1. $H^{*}\left(W\left(\mathfrak{a}_{n}\right), \mathfrak{g l}_{n}\right)=H^{*}\left(W\left(\mathfrak{g l}_{n}\right), \mathfrak{g l}_{n}\right)$.

The paper is organized as follows. In $\S 1$, we shall give the definitions of Weil algebra and some related structures. In $\S 2$, we shall consider the Lie algebra $a_{n}$ and formulate the main theorem. The proof of the main theorem will be given in $\S 3$. In $\S 4$, we shall give some remarks on $H^{*}(W(\mathfrak{g}), \mathfrak{h})$ for certain Lie algebra $\mathfrak{g}$ and subalgebra $\mathfrak{h}$.

\section{$\S 1$. Weil algebra}

Let $\mathfrak{g}$ be a topological Lie algebra over the real field $\boldsymbol{R}$. Recall that, for $p \geqq 0, q \geqq 0$, the $(p, q)$-cochains for $g$ are continuous $(p+q)$-linear functionals on $g$

Received July 29, 1977. 
such that the values of which $\omega\left(X_{1}, X_{2}, \cdots, X_{p} ; Y_{1}, Y_{2}, \cdots, Y_{q}\right)$ are skew-symmetric with respect to the permutations of $X_{1}, \cdots, X_{p}$ and symmetric with respect to the permutations of $Y_{1}, \cdots, Y_{q}$. These cochains make up a vector space $W^{p, q}(\mathfrak{g})$ over $\boldsymbol{R}$. We put $W^{0,0}(\mathfrak{g})=\boldsymbol{R}$.

We assign the space $W^{p, q}(\mathrm{~g})$ the degree $p+2 q$. Let $W^{s}(\mathrm{~g})=\underset{s=p+2 q}{\bigoplus} W^{p, q}(\mathrm{~g})$ and $W(\mathfrak{g})=\bigoplus_{s} W^{s}(\mathfrak{g})$.

We can canonically define a multiplication $W^{p, q}(\mathfrak{g}) \otimes W^{s, t}(\mathfrak{g}) \rightarrow W^{p+s, q+t}(\mathfrak{g})$. More precisely, for two cochains $\omega \in W^{p, q}(\mathrm{~g}), \eta \in W^{s, t}(\mathrm{~g})$, the product $\omega \eta \in$ $W^{p+s, q+t}(\mathrm{~g})$ is defined by the formula

$$
\begin{aligned}
(\omega \eta) & \left(X_{1}, \cdots, X_{p+s} ; Y_{1}, \cdots, Y_{q+t}\right) \\
= & \sum(-1)^{\imath_{1}+\cdots+\imath_{p}-(p+1) p / 2} \omega\left(X_{\imath_{1}}, \cdots, X_{\imath_{p}} ; Y_{k_{1}}, \cdots, Y_{k_{q}}\right) \times \\
& \times \eta\left(X_{\jmath_{1}}, \cdots, X_{\jmath_{s}} ; Y_{m_{1}}, \cdots, Y_{m_{t}}\right) .
\end{aligned}
$$

Here the summation is taken over all partitions of the sets $\{1, \cdots, p+s\}$ and $\{1, \cdots, q+t\}$ into disjoint subsets $\left\{\imath_{1}, \cdots, \imath_{p}\right\},\left\{\jmath_{1}, \cdots, \jmath_{s}\right\}$ with $\imath_{1}<\cdots<\imath_{p}, \jmath_{1}<\cdots$ $<\jmath_{s}$ and $\left\{k_{1}, \cdots, k_{q}\right\},\left\{m_{1}, \cdots, m_{t}\right\}$ with $k_{1}<\cdots<k_{q}, m_{1}<\cdots<m_{t}$ respectively. The cochain $\omega \eta$ is evidently continuous. With this multiplication, $W(\mathfrak{g})$ is a graded algebra over $\boldsymbol{R}$.

According to the usual way, to each $X \in \mathfrak{g}$, we can define the inner product $i(X)$, the infinitesimal transformation $\theta(X)$ and the differential $d$. For the sake of completeness, we give definitions.

Definttion of $i(X)$. For each $X \in \mathfrak{g}, i(X)$ is a linear map of $W^{p, q}(\mathfrak{g})$ into $W^{p-1, q}(\mathrm{~g})$ defined by the formula

$$
\begin{aligned}
& (i(X) \omega)\left(X_{1}, \cdots, X_{p-1} ; Y_{1}, \cdots, Y_{q}\right) \\
& =\omega\left(X, X_{1}, \cdots, X_{p-1} ; Y_{1}, \cdots, Y_{q}\right), \\
& \quad \text { for } \quad X_{\imath}, Y_{j} \in \mathfrak{g}, p>0, q \geqq 0 \text { and } \omega \in W^{p, q}(\mathfrak{g}) .
\end{aligned}
$$

If $p=0$, we put $i(X)\left(W^{0, q}(\mathrm{~g})\right)=0$.

Defintion of $\theta(X)$. For each $X \in \mathfrak{g}, \theta(X)$ is a linear map of $W^{p, q}(\mathfrak{g})$ into $W^{p, q}(\mathrm{~g})$ defined by the formula

$$
\begin{aligned}
& (\theta(X) \omega)\left(X_{1}, \cdots, X_{p} ; Y_{1}, \cdots, Y_{q}\right) \\
& =-\sum_{\imath} \omega\left(X_{1}, \cdots,\left[X, X_{\imath}\right], \cdots, X_{p} ; Y_{1}, \cdots, Y_{q}\right) \\
& \quad-\sum_{\jmath} \omega\left(X_{1}, \cdots, X_{p} ; Y_{1}, \cdots,\left[X, Y_{\jmath}\right], \cdots, Y_{q}\right), \\
& \quad \text { for } \quad X_{\imath}, Y_{j} \in \mathfrak{g}, p \geqq 0, q \geqq 0 \text { and } \omega \in W^{p, q}(\mathrm{~g}) .
\end{aligned}
$$

Definition of the differential $d$. We define the differentials $d_{1}: W^{p, q}(\mathfrak{g}) \rightarrow$ $W^{p+1, q}(\mathrm{~g})$ and $d_{2}: W^{p, q}(\mathrm{~g}) \rightarrow W^{p-1, q+1}(\mathrm{~g})$ by the following formulas. 
For $\alpha \in W^{0,0}(\mathfrak{g}), d_{1} \alpha=0, d_{2} \alpha=0$.

For $\omega \in W^{p, q}(\mathfrak{g}), p+q>0$,

$$
\begin{aligned}
& \left(d_{1} \omega\right)\left(X_{1}, \cdots, X_{p+1} ; Y_{1}, \cdots, Y_{q}\right) \\
& =\sum_{\imath<j}(-1)^{\imath+\jmath} \omega\left(\left[X_{\imath}, X_{\jmath}\right], X_{1}, \cdots, \hat{X}_{\imath}, \cdots, \hat{X}_{\jmath}, \cdots, X_{p+1} ; Y_{1}, \cdots, Y_{q}\right) \\
& \quad+\sum_{\imath, \lambda}(-1)^{\imath} \omega\left(X_{1}, \cdots, \hat{X}_{\imath}, \cdots, X_{p+1} ;\left[X_{\imath}, Y_{\lambda}\right], Y_{1}, \cdots, \hat{Y}_{\lambda}, \cdots, Y_{q}\right), \\
& \left(d_{2} \omega\right)\left(X_{1}, \cdots, X_{p-1} ; Y_{1}, \cdots, Y_{q+1}\right) \\
& \quad=\sum_{\imath} \omega\left(Y_{\imath}, X_{1}, \cdots, X_{p-1} ; Y_{1}, \cdots, \hat{Y}_{\imath}, \cdots, Y_{q+1}\right) .
\end{aligned}
$$

In these definitions, we note that the resulting cochains are really continuous.

$R E M A R K$. As to $d_{1}$ we note that if $p=0$ (resp. $q=0$ ), then the first (resp. the second) summation is defined to be zero. As to $d_{2}$, if $p=0$, then we put $d_{2}=0$.

The following assertion is verified by easy computations.

LEMMA 1.1. (i) $d_{1}{ }^{2}=0$, (ii) $d_{2}^{2}=0$, (iii) $d_{1} d_{2}+d_{2} d_{1}=0$.

Hence, putting $d=d_{1}+d_{2}$, we have $d^{2}=0$. Thus $d$ gives rise to a differential on $W(\mathrm{~g})$.

If $\mathrm{g}$ is finite-dimensional, the definitions of $i(X), \theta(X)$ and $d$ coincide with usual ones given by Cartan [1]. These operators are related to each other as follows.

LEMMA 1.2. For each $X \in g$, we have

(I) $\theta(X)=i(X) d+d i(X)$,

(II) $i([X, Y])=\theta(X) i(Y)-i(Y) \theta(X)$,

(III) $\theta([X, Y])=\theta(X) \theta(Y)-\theta(Y) \theta(X)$.

Moreover the multiplication in $W(\mathfrak{g})$ is related to the differential $d$ through the usual formula

(IV) $d(\omega \eta)=(d \omega) \eta+(-1)^{\text {degree } \omega} \omega(d \eta)$.

Proof. The formulas (I), (II) and (III) are verified by easy computations. According to the definition, we can establish the identity

$$
i(X)(\omega \eta)=(i(X) \omega) \eta+(-1)^{p} \omega(i(X) \eta), \text { for } \omega \in W^{p, q}(\mathfrak{g}), \eta \in W^{s, t}(\mathfrak{g}) .
$$

In other words, $i(X)$ is an antiderivation.

In order to show the formula (IV), we shall first show that the infinitesimal transformations are derivations. That is, 


$$
\theta(X)(\omega \eta)=(\theta(X) \omega) \eta+\omega(\theta(X) \eta), \text { for } \omega \in W^{p \cdot q}(\mathfrak{g}), \eta \in W^{s, t}(\mathfrak{g}) .
$$

We proceed by induction on the sum $p+s$. Let $\omega \in W^{0, q}(\mathrm{~g})$ and $\eta \in W^{0, t}(\mathrm{~g})$. Then we have, for $X, Y_{i} \in \mathrm{g}$,

$$
\begin{aligned}
\theta(X)(\omega \eta)\left(Y_{1}, \cdots, Y_{q+t}\right)=-\sum_{\jmath}(\omega \eta)\left(Y_{1}, \cdots,\left[X, Y_{\jmath}\right], \cdots, Y_{q+t}\right) \\
=-\sum_{\jmath}\left(\sum \omega\left(Y_{\imath_{1}}, \cdots,\left[X, Y_{\jmath}\right], \cdots, Y_{\imath_{q}}\right) \eta\left(Y_{\jmath_{1}}, \cdots, Y_{\jmath_{t}}\right)\right. \\
\left.+\sum \omega\left(Y_{k_{1}}, \cdots, Y_{k_{q}}\right) \eta\left(Y_{m_{1}}, \cdots,\left[X, Y_{\jmath}\right], \cdots, Y_{m_{t}}\right)\right) \\
=((\theta(X) \omega) \eta)\left(Y_{1}, \cdots, Y_{q+t}\right)+(\omega(\theta(X) \eta))\left(Y_{1}, \cdots, Y_{q+t}\right) .
\end{aligned}
$$

Now assuming that it holds in the case $p+s=r$, we prove that it holds in the case $p+s=r+1$. Let $\omega \in W^{p, q}(\mathrm{~g}), \eta \in W^{s, t}(\mathrm{~g})$ and let $p+s=r+1$. It is sufficient to show that, for any $X, Y \in \mathfrak{g}$,

$$
i(Y)(\theta(X)(\omega \eta))=i(Y)((\theta(X) \omega) \eta+\omega(\theta(X) \eta)) .
$$

Since $i(Y) \theta(X)=\theta(X) i(Y)-i([X, Y])$ by (II) and since the inner product is an antiderivation,

$$
\begin{aligned}
i(Y)(\theta(X)(\omega \eta))=\theta(X)(i(Y)(\omega \eta))-i([X, Y])(\omega \eta) & \\
=\theta(X)((i(Y) \omega) \eta & \left.+(-1)^{p} \omega(i(Y) \eta)\right) \\
- & \left((i([X, Y]) \omega) \eta+(-1)^{p} \omega(i([X, Y]) \eta)\right) .
\end{aligned}
$$

Then, by assumption, we see that

$$
\begin{aligned}
& \theta(X)(i(Y) \omega) \eta=(\theta(X) i(Y) \omega) \eta+(i(Y) \omega)(\theta(X) \eta), \\
& \theta(X)(\omega(i(Y) \eta))=(\theta(X) \omega)(i(Y) \eta)+\omega(\theta(X) i(Y) \eta) .
\end{aligned}
$$

Hence we have

$$
\begin{aligned}
i(Y)(\theta(X)(\omega \eta))=((\theta(X) i(Y)-i([X, Y])) \omega) \eta \\
\quad+(-1)^{p} \omega((\theta(X) i(Y)-i([X, Y])) \eta)+(i(Y) \omega)(\theta(X) \eta) \\
\quad+(-1)^{p}(\theta(X) \omega)(i(Y) \eta) \\
=(i(Y) \theta(X) \omega) \eta+(-1)^{p}(\theta(X) \omega)(i(Y) \eta)+(i(Y) \omega)(\theta(X) \eta) \\
\quad+(-1)^{p} \omega(i(Y) \theta(X) \eta) \\
=i(Y)((\theta(X) \omega) \eta+\omega(\theta(X) \eta)) .
\end{aligned}
$$

Thus the assertion is proved. That is, $\theta(X)$ is a derivation.

Now using the formula (I) and noting that $\theta(X)$ is a derivation and $i(X)$ is an antiderivation, we can similarly verify the formula (IV) by induction. 
q.e.d.

From lemma 1.2 , it follows that $W(\mathrm{~g})$ together with $d$ is a differential graded algebra over $\boldsymbol{R}$.

Definition. A differential graded algebra $W(\mathrm{~g})$ is called the Weil algebra of a topological Lie algebra $\mathrm{g}$.

We denote its $q$-th cohomology by $H^{q}(W(\mathfrak{g}))$ and its cohomology algebra by $H *(W(\mathfrak{g}))$.

The following is well-known in the case where $\mathfrak{g}$ is finitedimensional.

THEOREM 1.1. The cohomology of $W(\mathrm{~g})$ is trivial. That is, $H^{q}(W(\mathrm{~g}))=0$ for $q \geqq 1\left(\right.$ for $\left.q=0, H^{0}(W(\mathfrak{g}))=\boldsymbol{R}\right)$.

Proof. Let $\varepsilon$ be a mapping of $W(g)$ into itself defined by the formula, for $\omega \in W^{p, q}(\mathrm{~g})$,

$$
\begin{aligned}
& (\varepsilon \omega)\left(X_{1}, \cdots, X_{p+1} ; Y_{1}, \cdots, Y_{q-1}\right) \\
& \quad=\sum_{\imath}(-1)^{2} \omega\left(X_{1}, \cdots, \hat{X}_{\imath}, \cdots, X_{p+1} ; X_{\imath}, Y_{1}, \cdots, Y_{q-1}\right) .
\end{aligned}
$$

Then $\varepsilon$ is an antiderivation of degree -1 and we have:

$$
\begin{aligned}
& \left(\varepsilon d_{2}+d_{2} \varepsilon\right) \omega\left(X_{1}, \cdots, X_{p} ; Y_{1}, \cdots, Y_{q}\right) \\
& \quad=(p+q) \omega\left(X_{1}, \cdots, X_{p} ; Y_{1}, \cdots, Y_{q}\right) .
\end{aligned}
$$

We shall call the weight of an element of $W(\mathfrak{g})$ the largest integer $q$ such that its component in $\bigoplus_{p \geqq 0} W^{p, q}(\mathrm{~g})$ is not equal to zero. Let $\omega$ be a non-zero homogeneous element of degree $m(m \geqq 1)$ of $W(\mathrm{~g})$, then $d \varepsilon \omega+\varepsilon d \omega$ is homogeneous of degree $m$. Let $q \geqq 0$ be the weight of $\omega$. Then the weight of

$$
\eta=\omega-\frac{1}{(p+q)}(d \varepsilon+\varepsilon d) \omega
$$

is $\leqq q-1$. Now suppose that $\omega$ is a cocycle, then $\eta$ is a cocycle cohomologous to $\omega$ and has the weight strictly smaller than that of $\omega$. Repeating this argument, we see that $\omega$ is cohomologous to zero. This shows that $H^{m}(W(g))$ is trivial. q.e.d.

Let now $\mathfrak{h}$ be a subalgebra of $g$. We shall associate to $\mathfrak{h}$ some subalgebras of $W(\mathfrak{g})$. We say that an element of $W(\mathfrak{g})$ is mvariant with respect to $\mathfrak{h}$ or $\mathfrak{h}$ invariant if it is annihilated by all $\theta(X)$ for $X \in \mathfrak{h}$. Since the differential $d$ commutes with all infinitesimal transformations, the space $[W(\mathrm{~g})]^{\mathrm{h}}$ of all invariant cochains is a differential graded subalgebra of $W(\mathrm{~g})$. As the antiderivation $\varepsilon$ commutes with infinitesimal transformations, the cohomology $H^{*}\left([W(\mathfrak{g})]^{\text {h }}\right)$ of $[W(\mathrm{~g})]^{\mathrm{g}}$ is also trivial.

Now we say that a $\mathfrak{h}$-invariant element is basic with respect to $\mathfrak{h}$ or $\mathfrak{h}$-basic 
if it is annihilated by $i(X)$, for $X \in \mathfrak{h}$. We denote the space of all $\mathfrak{h}$-basic elements by $[[W(\mathrm{~g})]]^{\mathrm{h}}$. Clearly $[[W(\mathrm{~g})]]^{\mathrm{h}}$ is a differential graded subalgebra of $W(\mathrm{~g})$ by the formula (I). We use the notation $H^{*}(W(\mathfrak{g}), \mathfrak{h})$ for its cohomology algebra.

\section{$\S 2$. Main theorem}

In this section, $\mathfrak{a}_{n}$ denote the Lie algebra of formal vector fields at the origin in $\boldsymbol{R}^{n}$. The Krull topology turns $\mathfrak{a}_{n}$ into a topological Lie algebra over $\boldsymbol{R}$. Let $L_{k}$ be the set of all formal vector fields

$$
f_{1}\left(\partial / \partial x_{1}\right)+\cdots+f_{n}\left(\partial / \partial x_{n}\right),
$$

$f$, being a formal power series whose leading term is of degree $\geqq k+1$. Then $L_{k}$ is a subalgebra of $\mathfrak{a}_{n}$. Let $\mathfrak{g}^{2}$ be the set of all formal vector fields of the form (2.1), the $f_{j}$ 's being homogeneous polynomials of degree $\imath+1$. Then $L_{k}$ $=\mathrm{g}^{k} \oplus L_{k+1}$ and $\left[\mathrm{g}^{2}, \mathrm{~g}^{j}\right] \subset \mathrm{g}^{2+\jmath} . \mathrm{g}^{-1}$ is just $\boldsymbol{R}^{n}$ and $\mathrm{g}^{0}$ can be identified with the Lie algebra $\mathfrak{g l}_{n}$.

The topological dual space $a_{n}{ }^{\prime}$ is the direct sum

$$
\mathfrak{a}_{n}^{\prime}=\underset{\imath \geqq-1}{\bigoplus^{i}}\left(\mathfrak{g}^{i}{ }^{\prime} .\right.
$$

Moreover we have:

LEMMA 2.1. $W\left(\mathfrak{a}_{n}\right)$ is generated by $\mathfrak{a}_{n}{ }^{\prime}$ as an algebra over $\boldsymbol{R}$.

Proof. It is sufficient to show the following proposition.

Proposition. Let $\omega$ be a continuous q-linear form on $\mathfrak{a}_{n}$. Then for each $\imath=1, \cdots, q$, there exists some $k \geqq-1$ such that

$$
\omega \mid \mathfrak{a}_{n} \times \cdots \times \mathfrak{a}_{n} \times{ }^{\mathcal{j}} \bar{L}_{k} \hbar \times \mathfrak{a}_{n} \times \cdots \times \mathfrak{a}_{n} \equiv 0 .
$$

Proof. For the simplicity we assume $\jmath=1$. From the continuity of $\omega$, it follows that there exists some $k_{0}$ such that

$$
\omega \mid L_{k_{0}} \times \cdots \times L_{k_{0}} \equiv 0 \text {. }
$$

Let $\mathfrak{a}_{n}=N_{k_{0}}+L_{k_{0}}$, where $N_{k_{0}}$ is a complementary space to $L_{k_{0}}$. Then $N_{k_{0}}$ is a finite-dimensional vector space. Let $\left\{e_{1}, \cdots, e_{N}\right\}$ be a basis of $N_{k_{0}}$. Now consider the continuous $(q-1)$-linear forms on $\mathfrak{a}_{n}$ given by the formulas

$$
\omega_{\imath}\left(X_{1}, \cdots, X_{q-1}\right)=\omega\left(X_{1}, e_{\imath}, X_{\mathbf{2}}, \cdots, X_{q-1}\right), \imath=1, \cdots, N \text {. }
$$

By the continuity of $\omega_{i}$ and the finite-dimensionality of $N_{k_{0}}$, there exists some $k_{0}{ }^{\prime}$ such that

$$
\omega_{i} \mid L_{k_{0^{\prime}}} \times \cdots \times L_{k_{0^{\prime}}} \equiv 0 \text {, for any } \imath \text {. }
$$

Hence we have $\omega \mid L_{k_{0^{\prime}}} \times N_{k_{0}} \times L_{k_{0^{\prime}}} \times \cdots \times L_{k_{0^{\prime}}} \equiv 0$. Therefore, putting $k_{1}=\max \left(k_{0}\right.$, $k_{0}{ }^{\prime}$ ), we see that 


$$
\omega \mid L_{k_{1}} \times \mathfrak{a}_{n} \times L_{k_{1}} \times \cdots \times L_{k_{1}} \equiv 0 .
$$

The conclusion now follows by easy induction. Therefore lemma 2.1 is proved.

Since $\mathfrak{g l}_{n}$ is considered as a subalgebra of $\mathfrak{a}_{n}$, there exists a differential graded algebra homomorphism

$$
\varphi^{\prime}: W\left(\mathfrak{a}_{n}\right) \longrightarrow W\left(\mathfrak{g l}_{n}\right)
$$

and it induces

$$
\varphi:\left[\left[W\left(\mathfrak{a}_{n}\right)\right]\right]^{31 n} \longrightarrow\left[\left[W\left(\mathfrak{g l}_{n}\right)\right]\right]^{31 n} .
$$

Then we have the following main theorem and the proof will be given in the next section.

THEOREM 2.1. The homomorphism $\varphi$ of differentıal graded algebras induces an isomorphism on cohomology

$$
H^{*}\left(W\left(\mathfrak{a}_{n}\right), \mathfrak{g l}_{n}\right) \cong H *\left(W\left(\mathfrak{g l}_{n}\right), \mathfrak{g l}_{n}\right) .
$$

\section{§3. Proof of the main theorem}

We shall make use of the Hochschild-Serre spectral sequence associated to the subalgebra $\mathfrak{g l}_{n}$ of $\mathfrak{a}_{n}$.

Define a filtration in the complex $W\left(\mathfrak{a}_{n}\right)$ by the subspaces $A^{p}=\sum_{q} A^{p, q}$, where $A^{p, q}=W^{p+q}\left(\mathfrak{a}_{n}\right)$, for $p \leqq 0, A^{p, q}=0$, for $q \leqq 0$, and otherwise consists of all those cochains $\omega \in W^{p+q}\left(\mathfrak{a}_{n}\right)$ for which $i\left(X_{1}\right) \cdots i\left(X_{q+1}\right) \omega=0$ whenever $X_{1}, \cdots, X_{q+1} \in \mathfrak{g l}_{n}$. Each $A^{p, q}$ is naturally a topological $\mathrm{gl}_{n}$-module. We can show as in [3] that the $E_{1}{ }^{p, q}$-term of the spectral sequence corresponding to this filtration is isomorphic to $H^{q}\left(\mathfrak{g l}_{n} ; A^{p, 0}\right)$.

Now consider the radial vector field $R=\sum_{i} x_{i}\left(\partial / \partial x_{\imath}\right)$. Then making use of lemma 2.1, we can show that the $\mathfrak{g l}_{n}$-module $A^{p, 0}$ breaks up a sum $A^{p, 0}=\oplus V_{\lambda}{ }^{p}$ of eigenspaces of $\theta(R)$ with eigenvalues $\lambda \in \boldsymbol{R}$. Since $\mathfrak{g l}_{n}$ is reductive and the zero eigenspace $V_{0}{ }^{p}$ is finite-dimensional, $H^{q}\left(\mathfrak{g l}_{n} ; A^{p, 0}\right)=H^{q}\left(\mathfrak{g l}_{n} ;\left[A^{p, 0}\right]^{\mathfrak{g l} n}\right)$. Therefore,

$$
E_{1}^{p, q}=H^{p}\left(\mathfrak{g l}_{n} ; \boldsymbol{R}\right) \otimes\left[A^{p, 0}\right]^{g \gamma_{n}} .
$$

Clearly, $\left[A^{p, 0}\right]^{g 1 n}=\left[\left[W\left(\mathfrak{a}_{n}\right)\right]\right]^{g / n}$. Then it is easy to see that

$$
E_{2}^{p, q}=H^{q}\left(\mathfrak{g l}_{n} ; \boldsymbol{R}\right) \otimes H^{p}\left(W\left(\mathfrak{a}_{n}\right), \mathfrak{g l}_{n}\right) .
$$

Thus we have:

Proposition 3.1. There exists a spectral sequence $\left\{E_{r}{ }^{p, q}, d_{r}\right\}$ which converges to a graded module associated to $H *\left(W\left(a_{n}\right)\right)$. The $E_{2}$-terms of this spectal sequence have the form

$$
E_{2}^{p, q}=H^{q}\left(\mathfrak{g l}_{n}, \boldsymbol{R}\right) \otimes H^{p}\left(W\left(\mathfrak{a}_{n}\right), \mathfrak{g l}_{n}\right) .
$$

Since the above filtration is compatible with multiplication, i. e. if $\omega \in A^{p, q}$, $\eta \in A^{p^{\prime}, q^{\prime}}$, then $\omega \eta \in A^{p+q^{\prime}, q+q^{\prime}}$, the spectral sequence in proposition 3.1 is multipli- 
cative. Similarly we can obtain a spectral sequence $\left\{{ }^{\prime} E_{r}^{p, q}, d_{r}{ }^{\prime}\right\}$ which converges to a graded module associated to $H^{*}\left(W\left(\mathfrak{g l}_{n}\right)\right)$ and whose ' $E_{2}$-terms have the form

$$
{ }^{\prime} E_{2}{ }^{p, q}=H^{q}\left(\mathfrak{g l}_{n} ; \boldsymbol{R}\right) \otimes H^{p}\left(W\left(\mathfrak{g l}_{n}\right), \mathfrak{g l}_{n}\right) .
$$

As the graded algebra homomorphism $\varphi^{\prime}: W\left(\mathfrak{a}_{n}\right) \rightarrow W\left(\mathfrak{g l}_{n}\right)$ is compatible with the filtrations, it induces a homomorphism of spectral sequences.

In order to prove the main theorem, it is sufficient to show the following theorem.

THEOREM 3.1. $\varphi^{\prime}$ induces an ssomorphism of $E_{2}^{p, q}$ onto ' $E_{2}{ }^{p, q}$, for all $p \geqq 0$, $q \geqq 0$.

In order to prove this theorem, we shall summarize some basic facts about the spectral sequence $\left\{{ }^{\prime} E_{r}{ }^{p, q}, d_{r}{ }^{\prime}\right\}$.

The "zero column" ' $E_{2}{ }^{0, *}=\bigoplus_{q \geqslant 0}{ }^{\prime} E_{2}{ }^{0, q}=H^{*}\left(\mathfrak{g l}_{n} ; \boldsymbol{R}\right)$ is the exterior algebra in $h_{1}{ }^{\prime}, \cdots, h_{n}{ }^{\prime}$ over $\boldsymbol{R}$ with degree $h_{\imath}{ }^{\prime}=2 i-1$ and the "zero row" ' $E_{2}{ }^{*, 0}=\bigoplus_{p \geq 0}{ }^{\prime} E_{2}{ }^{p, 0}$ is the polynomial algebra in $C_{1}{ }^{\prime}, \cdots, C_{n}{ }^{\prime}$ with degree $C_{\imath}{ }^{\prime}=2 i$. Moreover we may take $C_{\imath}{ }^{\prime}(i=1, \cdots, n)$ so that $h_{\imath}{ }^{\prime}$ is mapped on $C_{\imath}{ }^{\prime}$ by $d_{2 i}{ }^{\prime}$.

Similarly $E_{2}{ }^{0, *}=H^{*}\left(\mathrm{gl}_{n} ; \boldsymbol{R}\right)$ is the exterior algebra in $h_{1}, \cdots, h_{n}$ with degree $h_{i}=2 i-1$. It is not difficult to see that $h_{\imath}$ is mapped on $h_{\imath}^{\prime}$ by the map induced from $\varphi^{\prime}$ in spectral sequences.

Proof of theorem 3.1. The first step. Let $x$ be a non-zero element of $E_{2}{ }^{1,0}$ $=H^{1}\left(W\left(\mathfrak{a}_{n}\right), \mathfrak{g l}_{n}\right)$. Then, for any $r \geqq 2, x$ is a $d_{r}$-cocycle and not a $d_{r}$-coboundary, because of the form of spectral sequence. This means that $E_{\infty}{ }^{1,0}$ is non-trivial. However $E_{\infty}{ }^{p, q}=0$, for $p+q>0$, because the cohomology of $W\left(\mathfrak{a}_{n}\right)$ is trivial. Hence $E_{2}{ }^{1,0}=H^{1}\left(W\left(\mathfrak{a}_{n}\right), \mathfrak{g l}_{n}\right)=0$. From this it follows that $E_{2}{ }^{1, q}=0$, for $q \geqq 0$. Now consider the element $h_{1} \in H^{1}\left(\mathfrak{g l}_{n}, \boldsymbol{R}\right)=E_{2}{ }^{0,1}$. If $d_{2} h_{1}=0$, then $d_{r} h_{1}=0$, for $r \geqq 2$. Therefore we have $E_{2}{ }^{0,1}=0$. But this is contrary to the acyclicity of $W\left(\mathfrak{a}_{n}\right)$. Hence $d_{2} h_{1}$ is non-zero in $E_{2}{ }^{0,1}$. Let $d_{2} h_{1}=C_{1}$. Then we can easily verify that $H^{1}\left(W\left(\mathfrak{a}_{n}\right)\right.$, $\left.\mathrm{gl}_{n}\right)=\boldsymbol{R} \cdot C_{1}$. Thus $\varphi^{\prime}$ induces an isomorphism of $E_{2}{ }^{p, q}$ onto ${ }^{\prime} E_{2}{ }^{p, q}$, for $q \geqq 0,2 \geqq p$ $\geqq 0$. Using this, we can easily see that $d_{2} h_{2}=0$, for $i \geqq 2$.

The second step. Let $n \geqq r \geqq 1$. Assume that $\varphi^{\prime}$ is an isomorphism of $E_{2}{ }^{p, q}$ onto ' $E_{2}{ }^{p, q}$, for $q \geqq 0$ and $2(r-1) \geqq p \geqq 0$. From this we can easily deduce the followings ;

(i) for $r-1 \geqq i \geqq 0, h_{\imath}$ is mapped on $C_{\imath}$ by the differential

$$
d_{2 \imath}: E_{2 \imath}{ }^{0,2 \imath-1} \longrightarrow E_{2 \imath}^{22,0} \text {; }
$$

(ii) $\varphi\left(C_{\imath}\right)=C_{\imath}{ }^{\prime}$;

(iii) for $r-1 \geqq i \geqq 1$ and $n \geqq j \geqq r, d_{2 i} h_{j}=0$. Using these facts, we shall prove the following lemma.

LEMMA. $\quad H^{2 r-1}\left(W\left(\mathfrak{a}_{n}\right), \mathfrak{g l}_{n}\right)=0$.

Proof. Let $x$ be a non-zero element of $H^{2 r-1}\left(W\left(\mathfrak{a}_{n}\right), \mathfrak{g l}_{n}\right)=E_{2}{ }^{2 r-1,0}$, then there 
exists an integer $s$ with $2 r-2 \geqq s \geqq 1$ and exists an element $y$ of $E_{s}{ }^{2 r-s-1, s-1}$ such that $d_{s} y=x$, because $E_{\infty}{ }^{2 r-1,0}=0$.

From the assumption it follows that if $s$ is even, then $E_{s}{ }^{2 r-1, s-1}=0$. Therefore $s$ must be odd. By proposition 3.1, $E_{2}{ }^{2 r-s-1, s-1}$ is generated by the elements of the form $h_{\imath_{1}} \cdots h_{\imath_{k}} C_{\jmath_{1}} \cdots C_{\jmath_{m}}$ with $k \geqq 1, \imath_{1}<\cdots<\imath_{k}, 2\left(\imath_{1}+\cdots+\imath_{k}\right)-k=s-1$ and $\jmath_{1} \leqq \cdots \leqq j_{m}, 2\left(\jmath_{1}+\cdots+\jmath_{m}\right)=2 r-s-1$. If $2 \imath_{1}, \cdots, 2 \imath_{k}>s$, then $2\left(\imath_{1}+\cdots+\imath_{k}\right)-k>s k$ $-k=k(s-1) \geqq s-1$. Therefore we may assume that $2 \imath_{1} \leqq s$. But $s$ is odd, we assume that $2 \imath_{1}<s$. In $E_{2 \imath_{1}}$-terms, $d_{2 \imath_{1}}\left(h_{\imath_{1}} \cdots h_{\imath_{k}} C_{\jmath_{1}} \cdots C_{\jmath_{m}}\right)=h_{\imath_{2}} \cdots h_{\imath_{k}} C_{\imath_{1}} C_{\jmath_{1}} \cdots C_{\jmath_{m}}$ is non-zero, because $h_{\imath_{1}}{ }^{\prime} \cdots h_{\imath_{k}}{ }^{\prime} \otimes C_{\imath_{1}}{ }^{\prime} C_{\jmath_{1}}{ }^{\prime} \cdots C_{\jmath_{m}}{ }^{\prime}$ is non-zero in ' $E_{2 \imath_{1}}$-terms. Since $2 i_{1}+1 \leqq s$, this shows that $E_{s}{ }^{2 r-s-1, s-1}$ is trivial. Then the lemma follows immediately.

Now consider $h_{r} \in E_{2}{ }^{0,2 r-1}=H^{2 r-1}\left(\mathfrak{g l}_{n} ; \boldsymbol{R}\right)$. By (iii) $h_{r}$ is a cocycle of $E_{2 r-1}{ }^{0,2 r-1}$. Hence, if $d_{2 r} h_{r}=0$, then $h_{r}=0$ in $E_{2 r}{ }^{0,2 r-1}$. Then $E_{\infty}{ }^{0,2 r-1}$ contains a non-zero element $h_{r}$. However, $E_{\infty}{ }^{p, q}=0$, for $p+q>0$. Therefore $d_{2 r} h_{r}=C_{r}$ must be nonzero in $E_{2 r}{ }^{2 r, 0}$. We may take $C_{r}$ so that $\varphi\left(C_{r}\right)=C_{r}{ }^{\prime}$.

Since $\varphi^{\prime}$ is a graded algebra homomorphism, it is not difficult to see that $\left(\boldsymbol{R}\left[C_{1}, \cdots, C_{r}\right]\right)^{r}=H^{2 r}\left(W\left(\mathfrak{a}_{n}\right), \mathfrak{g l}_{n}\right)$, here $\left(\boldsymbol{R}\left[C_{1}, \cdots, C_{r}\right]\right)^{r}$ denotes the set of all homogeneous polynomials in $C_{1}, \cdots, C_{r}$ of degree $2 r$. Thus we have an isomorphism

$$
E_{2}{ }^{p, q} \cong^{\prime} E_{2}{ }^{p, q} \text {, for } q \geqq 0 \text { and } 2 r \geqq p \geqq 0 .
$$

The third step. Repeating the above consideration, we get to $r=n$. Recall that ' $E_{2}{ }^{p, q}$ is generated by $\underset{q \leq 2 n-1}{\bigoplus^{\prime}} E_{2}{ }^{0, q}$ and $\bigoplus_{p \leq 2 n}{ }^{\prime} E_{2}{ }^{p, 0}$. That is, it is generated by $h_{1}{ }^{\prime}, \cdots, h_{n}{ }^{\prime}$ and $C_{1}{ }^{\prime}, \cdots, C_{n}{ }^{\prime}$. Moreover $\varphi^{\prime}\left(C_{\imath}\right)=C_{\imath}{ }^{\prime}$ and $\varphi^{\prime}\left(h_{\jmath}\right)=h_{\jmath}{ }^{\prime}$. Then it is not difficult to see that $\varphi^{\prime}$ is an isomorphism of $E_{2}{ }^{p, q}$ onto ${ }^{\prime} E_{2}{ }^{p, q}$. This completes the proof of theorem 3.1, hence of the main theorem.

\section{§4. Some remarks}

In general, when we study the cohomology of the subalgebra of $W(\mathrm{~g})$ consisting of certain basic elements, there might be two directions. In the first, we consider the basic elements of $W(\mathfrak{g})$ with respect to $g$ itself. In this direction, we have some examples in our context.

Consider the Lie algebra $\mathfrak{a}_{n}$. Recall that $\mathfrak{a}_{n}$ has a subalgebra $\mathfrak{g}^{-1}=\boldsymbol{R}^{n}$ and $\left[\mathfrak{g}^{-1}, \mathfrak{g}^{i}\right] \subset \mathfrak{g}^{2-1}$, for any $i$. Then making use of lemma 2.1 , we can verify the next proposition.

Proposition 4.1. $\left[\left[W\left(\mathfrak{a}_{n}\right)\right]\right]^{a_{n}}=0$.

This proposition asserts that the Lie algebra $\mathfrak{a}_{n}$ has no continuous invariant symmetric forms.

On the other hand, some Lie algebras have such forms. For instance, we have an isomorphism

$$
\left[\left[W\left(L_{0}\right)\right]\right]^{L_{0}}=\left[\left[W\left(\mathfrak{g l}_{n}\right)\right]\right]^{g 1 n},
$$


because $\mathrm{gl}_{n}$ is a subalgebra of $L_{0}$ and the natural projection of $L_{0}$ onto $\mathrm{gl}_{n}$ is a Lie algebra homomorphism.

In the second direction, we consider the basic elements of $W(\mathfrak{g})$ with respect to a proper subalgebra of $\mathrm{g}$. As an example, we have:

Proposition 4.2. $\left[\left[W\left(\mathfrak{a}_{n}\right)\right]\right]^{L_{0}}=0$.

This proposition follows from lemma 2.1 and the fact that $\left[\mathfrak{g}^{-1}, L_{0}\right]=\mathfrak{a}_{n}$.

However, for $k \geqq 1,\left[\left[W\left(\mathfrak{a}_{n}\right)\right]\right]^{L_{k}} \neq 0$.

We shall give another example. Define the Lie algebra $\mathfrak{a}_{n}{ }^{F}$ of formal differential operators of order one as the direct sum of the space $F_{n}$ of formal power series and $\mathfrak{a}_{n}$ with the bracket operation

$$
\left[f \oplus X, f^{\prime} \oplus X^{\prime}\right]=\left(X f^{\prime}-X^{\prime} f\right) \oplus\left[X, X^{\prime}\right],
$$

where $f, f^{\prime} \in F_{n}$ and $X, X^{\prime} \in \mathfrak{a}_{n}$. The natural topology in $\mathfrak{a}_{n}{ }^{F}$ turns it into a topological Lie algebra.

The argument in Section 3 can be applied to this case and the corresponding result is obtained.

Proposition 4.3. The cohomology algebra $H^{*}\left(W\left(\mathfrak{a}_{n}{ }^{F}\right)\right.$, $\left.\mathfrak{g l}_{n}\right)$ is isomorphic to $H *\left(W\left(\mathfrak{g l}_{n}\right), \mathfrak{g l}_{n}\right)$.

\section{REFERENCES}

[1] H. CARTAN, Notion d'algèbres differentielles; applications aux groupes de Lie et aux variétés où operent un groupe de Lie, Colloque de Topologie, Bruxelles (1950), 15-27.

[2] C. Godbillon, Cohomologies d'algèbres de Lie de champs de vecteurs formels, Séminaire Bourbaki (novembre 1972), exposé 421.

[3] G. Hochschild AND J. Serre, Cohomology of Lie algebras, Ann. of Math., 57 (1953), 591-603. 